\title{
JAN RÜDIGER
}

\section{Thalassocraties médiévales Pour une histoire politique des espaces maritimes}

Y a-t-il eu des thalassocraties au Moyen Âge? Nous approcherons notre sujet à travers un exemple tiré non de la Méditerranée, mais d'une de ces »autres Méditerranées du Nord «, chères à Fernand Braudel ${ }^{1}$. C'est un récit médiéval, le récit d'un passé maritime. Vers le milieu du XII ${ }^{\mathrm{e}}$ siècle, un chroniqueur du clergé de la cathédrale de Roskilde, sur l'île de Zélande, raconte la préhistoire de l'actuel Danemark, royaume alors en pleine transformation, en phase de devenir une monarchie seuropéenne ২. Parmi les histoires que le chroniqueur raconte, et qui constituent un fond de première importance pour la mythographie scandinave, figure le récit de la fondation même de la future ville épiscopale, nommée Roskilde d'après le nom du fondateur, le roi Ro, et celui des sources (kilde), fort abondantes en ce lieu. Ce roi Ro avait deux fils auxquels il allait laisser son royaume. À l'un d'eux, nous dit la chronique, il laisserait toute la terre ferme, et à l'autre, la mer: »alter terras, mare possedit alter « ${ }^{2}$.

Nous nous étonnons de ce partage, à première vue si grotesquement inégal. Mais rien dans le texte ne nous permet de penser que l'auteur ait voulu donner l'impression que Helgi, le rex marinus, ait été la victime d'un mauvais tour. Le public du haut Moyen Âge l'acceptait en tant que partage égal. Cela nous invite à réfléchir sur la façon de s'imaginer cela: un royaume fait d'eau.

L'ethnologie utilise le terme de »sociétés côtières« pour désigner les spécificités sociales de ces gens dont les bases matérielles et l'horizon mental sont circonscrits par la vie qui a le dos tourné à la terre et le regard porté vers la mer. Les grands imaginaires de l'Égée antique sont profondément marqués par cette vision. Il ne serait venu à l'esprit d'aucun auditeur de l'»Odyssée« de penser que le grand voyageur, persécuté par la colère du dieu, aurait pu tout simplement continuer son chemin à terre - là où, au contraire, il n'y avait que quelques pas jusqu'au monde inconnu des géants et des étrangers. Les peines de la troupe athénienne perdue à l'intérieur de l'Anatolie, racontées par Xénophone dans l'»Anabase«, prirent fin aussitôt que les Grecs purent s'exclamer, depuis la dernière colline: »thálassa! thálassa! «3 - la mer! À cette époquelà, Athènes était, depuis un certain temps déjà, solidement établie comme première puissance en Égée, ayant des visées sur la Méditerranée occidentale, tandis que le territoire contrôlé par la cité était de quantité presque négligeable. C'était, pour ainsi dire,

1 Fernand BRAUdel, La Méditerranée et le monde méditerranéen à l'époque de Philippe II, vol. 1 , Paris ${ }^{2} 1966$, p. 143.

2 Chronique de Lejre, chap. 3, éd. Matthias Claudius GERTZ, Copenhague 1917 (Scriptores minores historiae danicae medii aevi, 1), p. 34-53.

3 Xénophone, Anabase, 4,7,24; pour l'histoire ultérieure de ce cri voir Tim Rood, The Sea! The Sea! The Shout of the Ten Thousand in the Modern Imagination, Londres 2004. 
tout juste le morceau de terre qu'il fallait pour y construire des maisons et des temples. Athènes se contentait d'une sorte de szone de trois milles`sur terre; la vraie superficie d'Athènes et de sa Ligue de Délos - dont les membres étaient eux aussi presque tous quasiment sans territoire - était la surface de la mer. C'était l'inversion de nos idées de territorialité. La partie du monde qui pouvait être contrôlée, décrite, vécue, était la partie couverte d'eau. Ce qui faisait la force d'Athènes, c'était son >maritoire « - mot insolite il est vrai, mais le fait qu'il n'existe pas en dit long sur nos conventions de nous représenter l'organisation politique de la surface de la planète. Elle correspond pourtant à une certaine vision de la réalité sociale. Lorsque Platon s'imagina la »Politeia« parfaite, loin des influences corrompues, elle ne s'érigeait point sur cette »île lointaine« des proverbes, mais, au contraire, dans les profondeurs du continent sans port ou autre accès à la mer ${ }^{4}$.

En même temps, les Athéniens s'étaient mis à réfléchir sur les conditions pour ainsi dire >réelles`, politiques, de leur prépondérance maritime. Hérodote, auquel les Anglais doivent le mot des »murailles de bois« si souvent invoqué à l'époque moderne 5 , employa - ou bien créa - le verbe »thalassokratéō«, dérivé de »thálassa « (la mer) et de ce terme qui se trouve au cœur de la pensée politique des Grecs, »kratéein« (dominer, contrôler, donner de l'ordre social). À l'époque romaine, au temps de Jules César, Strabon emploie le substantif abstrait »thalassokratía « ${ }^{6}$. Cette Rome jadis agraire et repliée sur le Latium s'était alors depuis longtemps faite l'héritière de Carthage et des grandes villes de l'Orient. De plus, elle venait de sortir de quelques guerres acharnées contre les >pirates`, se rendant ainsi compte que le contrôle de la Méditerranée était d'une importance primordiale. Contrairement à nos idées reçues, contrairement surtout à sa propre image d'elle-même ${ }^{7}$, Rome fut la plus grande des thalassocraties du monde ancien.

En grec moderne, le terme »thalassocratie« est toujours vivant; on l'utilise par exemple pour désigner 1'Empire britannique ${ }^{8}$. Tout comme le monde antique, l'époque moderne elle aussi paraît très océanique à notre conscience historique: Vasco da Gama, l'Armada, le capitaine Cook, les Compagnies des Indes et les navires des flottes britanniques puis américaines, et ainsi de suite jusqu'à la crise de Suez au moins. Les grandes puissances de l'époque moderne sont presque toutes transmarines; enfin, n'estce pas le début de l'expansion européenne sur les océans qui marque conventionnellement le début même de l'époque moderne? Face à ces »grands récits«, encore une fois,

4 Selon Strabon (Géographie, VII 3,8), Platon aurait cautionné contre un emplacement proche de la mer ponērodidáskalos (enseignante de vices), ce qui a donné à Peregrine HORDEN et Nicholas PURCELl le thème de leur œuvre magistrale: IDD., The Corrupting Sea. A Study of Mediterranean History, Oxford 2001.

5 Fameux grâce au garde du Grand Sceau Thomas Coventry, $1^{\text {er }}$ baron Coventry, dans une adresse au Collège des Juges de Londres, en 1634.

6 Strabon, Géographie, I 3,2 (en parlant de la Crète minoenne); voir les références dans Henry Georges LidDELL, Robert ScOTT, A Greek-English Lexicon, Oxford ${ }^{9} 1940$, s. v. thalassokratéo.

7 Depuis l'image des jumeaux fondateurs la main à l'araire jusqu'aux fantaisies bucoliques de l'ère impériale, la rus n'a-t-elle pas toujours été l'idéal proclamé de la >vraie〈 Rome?

8 Méga léxikon tēs hellēnikēs glōssēes, Athènes, Thessalonique 1930, s. v. thalassokratía. 
le Moyen Âge joue le rôle d'époque sentre-deux`, déficitaire. Ses figures emblématiques ne sont pas Ulysse, Énée et Octavien sur sa trirème devant Actium, ni Jacques Cartier ou l'amiral Nelson. Ce sont le laboureur avec l'araire ou la charrue; le seigneur sur sa motte et le châtelain dans son donjon s'appliquant à recouvrer les devoirs payés en grain ou en fruits; les rois et leur cortège sur chemins mouillés; et ces moines défricheurs, chers à Jacques Le Goff, qui s'en prennent au sous-bois. En somme, le Moyen Âge donne l'impression d'être la seule époque de l'histoire européenne qui soit presque uniquement terrienne. ${ }^{9}$

Comment alors écrire une histoire politique des espaces maritimes au Moyen Âge? Le mot »thalassocratie« pourra nous servir pour désigner les formes et modalités de domination sur les espaces maritimes. Or, comment l'employer? À mon avis, deux possibilités se dessinent. L'une est descriptive, portant le regard sur les formations politiques étroitement liées au pouvoir marin: par exemple, le conjoint anglo-danonorvégien sous Knut le Grand qui, en effet, fait de la mer du Nord le centre de son empire au début du $\mathrm{XI}^{\mathrm{e}}$ siècle, ou bien, en Méditerranée, la Couronne d'Aragon au $\mathrm{XIV}^{\mathrm{e}}$ siècle, en passant par Pise, la Sicile islamique et même, peut-être, l'Empire byzantin à certains moments de son histoire ${ }^{10}$. Non que leur histoire ne soit depuis longtemps étudiée par les historiens; mais on conviendra que face aux images d'Épinal de l'histoire médiévale - le laboureur, le seigneur et le moine défricheur - ces régions plutôt maritimes de l'Europe restent toujours un peu à l'écart, à la périphérie d'un continent dont le centre se trouve, on le dit souvent, entre la Loire, le Rhin et les Alpes ${ }^{11}$. Mettre, en revanche, en relief cette autre Europe, l'Europe maritime, avec le regard du comparatiste, n'apporterait pas seulement de nouvelles perspectives sur chacune de ces régions - pourquoi pas des études comparatives suivies des détroits du Danemark avec le détroit d'Otrante ou le Bosphore, des archipels baltiques et atlantiques avec l'Égée? On trouverait autant de différences que de ressemblances entre la Méditerranée et les »Méditerranées du Nord«, mais peu importe: la valeur du comparatisme, tel que Marc

9 L'Europe maritime n'y figure, en somme, que comme un »autre«, d'où partent des incursions (de Normands, de pirates musulmans...) qui affectent l'Europe des rois, des paysans et des défricheurs. Il n'y a guère de manuel de l'histoire médiévale qui traite d'un roi viking ou d'un port littoral du Portugal ou de l'Adriatique comme phénomène représentatif de l'ensemble de l'Europe, tel qu'il est très souvent le cas pour les rois ou les villages français et allemands.

10 Parmi toutes les œuvres vouées à ce sujet, citons le tome dans la série Faire l'Europe: Michel Mollat, L'Europe et la mer, Paris 1999, en voyant bien sûr, ce qui dépasse l'époque médiévale. Sauf erreur, le premier médiéviste qui se soit servi du terme »thalassocratie« fut Archibald Lewis, en parlant des Celtes des $V^{\mathrm{e}}-\mathrm{VIII}^{\mathrm{e}}$ siècles: ID., The Northern Seas. Shipping and Commerce in Northern Europe, AD 300-1100, Princeton 1958, p. 64.

11 À titre indicatif, voir Michael MITTERAUER, Warum Europa? Mittelalterliche Grundlagen eines Sonderwegs, Munich 2003, p. 17. Mais la tendance à y voir le centre dynamique marque bien d'autres grands récits depuis Jacques LE GOFF, La civilisation de l'Occident médiéval, Paris 1964 (qui n'était certainement pas le premier), en passant par Georges DuBY, L'Europe des cathédrales. L'art et la société 980-1420, Paris 1980, jusqu'à Robert BARTLETT, The Making of Europe. Conquest, Colonization and Cultural Change, Londres 1993, qui ont tous eu une influence considérable sur la recherche médiévistique dans son ensemble. On pourrait y ajouter nombre d'œuvres d'une tendance semblable mais qui ont moins de portée. 
Bloch nous l'a enseigné ${ }^{2}$, ne réside-t-elle pas précisément dans la possibilité de faire apparaître aussi bien les phénomènes qui sont pareils que ceux qui ne le sont pas? Bien davantage, étudier les différentes périphéries de l'Europe terrienne les ferait apparaître enfin moins >périphériquesく. Les spécialistes des mers du Nord et ceux de la Méditerranée, tous un peu sur la défensive devant le poids écrasant de l'Europe franque dans l'image collective du Moyen Âge, se retrouveraient ainsi dans un effort commun pour redresser un peu le bilan. Peut-être le terme »thalassocratie« fournit-il le tertium nécessaire pour que la comparaison historique puisse se faire. Il serait alors un outil intellectuel qui servirait à mettre en œuvre la comparaison.

L'autre emploi du terme, nous l'appellerons sanalytique`, va encore un peu plus loin. Plutôt que de désigner des formations politiques à caractère maritime, le terme $»$ thalassocratie« servirait alors à poser la question de savoir s'il peut exister un pouvoir fondé essentiellement sur la mer, et quelles formes il revêtirait. Cette stratégie de recherche, si elle va au-delà de la description comparatiste, est aussi plus étroite: le terme »thalassocratie« porterait désormais exclusivement sur les formes de domination de l'espace maritime qui, du fait d'être basées sur la mer, sont fondamentalement différentes de celles qui sont principalement basées sur la terre ferme. (Voici d'ailleurs une métaphore mal tournée: l'image du pouvoir qui doit toujours être >basé« sur quelque chose de ferme et solide en dit long sur nos idées.)

Dans ce sens, le terme s'appliquerait avec beaucoup de réserves. Toutes les puissances ayant des navires ne sont pas nécessairement des thalassocraties. Prenons quelques exemples tirés de l'histoire moderne et contemporaine pour ne pas anticiper le débat des médiévistes: les États-Unis actuels ne sont pas qualifiés de thalassocratie, bien qu'ils soient peut-être la plus grande puissance navale de l'histoire. Mais ce n'est ni la Navy ni le contrôle sur les routes maritimes qui font la base de la puissance américaine; c'est plutôt - mises à part la culture et l'idéologie - le contrôle d'un vaste territoire, bientôt dépeuplé de ses indigènes, très fertile, et, pendant longtemps, hautement technisé. Si l'on applique une définition très étroite, l'Empire britannique lui-même ne serait pas une vraie thalassocratie, car c'est d'abord l'efficacité de son économie agraire qui a fait la force de l'Angleterre du XV siècle jusqu'à l'industrialisation. Les Pays-Bas du XVII ${ }^{\mathrm{e}}$ siècle sont peut-être un cas à la limite, vu que la plupart de leurs états, bien que très peuplés et bien aménagés, ne sont vraiment pas à la base de la puissance maritime néerlandaise qui a été avant tout le projet de l'oligarchie mercantile de quelques villes de Hollande. Mais la puissance que les Hollandais exerçaient de fait portait sur les îles de l'Inde, et non pas sur les routes maritimes dont ils souhaitaient, en revanche, qu'elles soient libres de toute domination politique - le mare liberum de Grotius.

Qu'en est-il de Venise? Sans doute est-ce une formation politique dont l'appui territorial est négligeable, presque inexistant. Aucune pratiquement des conquêtes ultérieures de la République ne visèrent le contrôle des arrière-pays des points d'appui

12 Son intervention au Congrès international des sciences historiques (Oslo, 1928) reste le document fondateur du comparatisme en histoire: Marc BLOCH, Pour une histoire comparée des sociétés européennes, dans: ID., Mélanges historiques, Paris 1963, p. 16-40. 
conquis. L'objectif était plutôt les routes maritimes qui les reliaient. L'expansion dans le Veneto se fit plusieurs siècles après que Venise fut devenue assez puissante pour conquérir Constantinople et bien d'autres lieux et recoins lointains de la Méditerranée. Vraiment lointains? Évidemment, pendant longtemps Raguse et Candie paraissaient aux Vénitiens moins >lointaines` que Vérone. Les distances se mesurent d'une manière tout à fait différente selon que l'on habite la mer ou la terre. Si Venise constitue donc un cas de thalassocratie dans le sens stricte du terme - hypothèse à discuter! -, elle pose une question tout à fait intéressante. L'emprise vénitienne sur son propre arrièrepays coïncide, on le sait d'après les faits, avec le début du déclin prolongé de la puissance méditerranéenne de la République. Alors, pour une thalassocratie, l'acquisition d'une terra ferma ne constituerait pas un renforcement, mais une hypothèque avec de graves répercussions?

Si l'on prend le terme »thalassocratie« pour désigner une culture politique spécifiquement maritime, quelles en seraient les traits caractéristiques? Un proverbe qui existe en plusieurs langues, dont l'allemand, dit: »l'eau n'a pas de poutres «13. Il faut s'en méfier, on ne peut pas s'y appuyer. Le proverbe a une longue histoire; c'est un des Sept Sages de l'Antiquité grecque, Pittakos de Mytilène, qui le formula ainsi: »pistòn gē, ápiston thálassa «14. La mer se dérobe au calcul, à la prévisibilité, et enfin, à la structure. C'est à raison que >fluideく est une métaphore prisée des historiens des sociétés sans État ${ }^{15}$. Pour la thalassocratie en tant que forme politique, cette observation est fondamentale: il est tout simplement impossible de répéter sur la mer ce qu'il est convenu d'appeler l'>encadrement` des campagnes (féodal, paroissial, etc.). Au cours du Moyen Âge central, au premier temps féodal, les populations sont conscientes du fait qu'elles vivent dans un certain endroit et doivent tel cens et tel service à tel seigneur. Elles ont raison d'espérer des miracles de tel saint parce qu'il veille sur l'ouaille de telle église et parce qu'elles doivent payer la dîme à tel prêtre en reconnaissance. Audelà de cette forêt ou ce ruisseau, ce serait un autre prêtre et un autre saint. Il y a des lieux sur les routes où le péage est perçu ${ }^{16}$. Sur la mer, on ne construit pas de rues et on ne prélève pas de péages, du moins pas à des lieux fixes ni de manière régularisée. Enfin, l'histoire du sundtold, le péage des détroits danois ${ }^{17}$, montre qu'il est assez dif-

13 »Wasser hat keine Balken «.

14 Rapporté par, entre autres, Diogène Laërce, Vitae philosophorum I 77, éd. Miroslav MARCOVICH, Stuttgart, Leipzig 1999, p. 53-54; voir Gerhard FINK, Die griechische Sprache, Munich 1992, p. 25-27.

15 Pour la critique de ce parti pris, voir Georges BALANDIER, Anthropologie politique, Paris 1967, toujours utile.

16 Pour ce tableau, voir les synthèses bâties sur les grandes monographies régionales des années 1970-1980, telles Robert FOSSIER, Enfance de l'Europe, $\mathrm{X}^{\mathrm{e}}-\mathrm{XII}^{\mathrm{e}}$ siècle. Aspects économiques et sociaux, 2 vol., Paris 1982 (Nouvelle Clio. L'histoire et ses problèmes, 17-17bis); ID., La société médiévale, Paris 1991; Jean-Pierre POLY, Éric BOURNAZEL, La mutation féodale, $\mathrm{X}^{\mathrm{e}}-$ $\mathrm{XII}^{\mathrm{e}}$ siècle, Paris 1980 (Nouvelle Clio. L'histoire et ses problèmes, 16); Dominique BARTHÉLEMY, L'ordre seigneurial $\left(\mathrm{XI}^{\mathrm{e}}-\mathrm{XII}^{\mathrm{e}}\right.$ siècles), Paris 1990.

17 Voir, à côté de toute une littérature spécialisée en langues scandinaves et en allemand, Nils Hybel, Bjørn Poulsen, The Danish Resources c. 1000-1550, Leyde 2007 (The Northern World, 34). 
ficile de le prélever même aux rares endroits où la géographie permet de le faire. On ne peut pas dresser de frontières; on ne peut même pas les penser. On ne peut pas construire de châteaux forts: même les plus lourds navires de guerre sont quelque chose de tout à fait différent. Le passage des sociétés occidentales, des structures migrantes aux structures fixes, immobiles, a commencé avec l'agriculture et s'est achevé quand le transfert régulier de villages prit fin au haut Moyen Âge - ce changement, bien que fondamental pour l'histoire continentale, n'a pas d'équivalent en Europe maritime. Enfin les structures sociales, le monde mental, ce que la nouvelle histoire culturelle appelle le »sense of space«, tout cela s'est de plus en plus développé en différentes directions et a fait des zones maritimes des endroits de plus en plus caractéristiques puis marginalisés, jusqu'à ce que les révolutions de l'âge industriel, infrastructurelles et culturelles, y aient mis fin.

Évidemment, tout cela a de graves conséquences pour l'exercice du pouvoir. Un château fort peut servir à son détenteur pendant des siècles, du moins si on a de la chance et qu'il ne s'agit pas de Château-Gaillard. En revanche, même la flotte la plus puissante ne peut contrôler un certain espace avec la même régularité. Il est vrai qu'au haut Moyen Âge, le pouvoir politique sur terre n'était pas lui non plus tellement immobile. Mais il y a une grande différence entre la fluidité thalassocratique et la royauté itinérante, même à l'époque carolingienne ou à celles des premiers Capétiens et Ottoniens. La royauté itinérante n'est pas vraiment ambulante mais plutôt décentrée; elle oscille entre un nombre de points d'appui fixes selon les nécessités de l'alimentation et de la présence dans les régions. Bien aménagé, un tel système pouvait tenir pendant de longues périodes. À l'opposé, la perte de quelques positions centrales aurait amené un affaiblissement sérieux ou même la fin du règne.

Il en va tout autrement en mer. D'abord, il n'y a pas de ressources sur lesquelles on peut s'appuyer avec la même assurance que les mines d'argent ottoniennes. Même les produits agricoles, toujours menacés de mauvaises récoltes, demeurent plus fiables. C'était déjà le comble si, pendant un certain temps, on pouvait compter sur les bancs de harengs dans la Baltique ${ }^{18}$. La vie sur mer, l'exercice de pouvoir sur la mer présupposent qu'on tienne compte de la contingence. On ne peut guère échapper à la contingence, ce que les rois et d'autres grands personnages du haut Moyen Âge - pour ne pas parler des époques plus récentes - avaient au moins tenté de faire en s'établissant dans des régions centrales, tels le Harz ou le Bassin parisien. En fin de compte, un thalassocrate ne peut se replier nulle part. Les pirates, dans la mer Baltique et sans doute ailleurs, pouvaient en une seule nuit franchir la mer, gagner une ville portuaire et renverser la balance du pouvoir maritime. Ces mêmes pirates ont dû apprendre à leurs dépens que leur système de lieux de repli - système sûrement aussi développé que possible dans les circonstances du moment - devait perdre toute sa valeur lors d'un seul contre-

18 Voir Carsten JAHnKe, Das Silber des Meeres. Fang und Vertrieb von Ostseeheringen zwischen Norwegen und Italien (12.-16. Jahrhundert), Cologne 2000 (Quellen und Darstellungen zur hansischen Geschichte, nouv. sér. 49). 
vent ${ }^{19}$. On dira peut-être qu'il existe néanmoins les ports. L'histoire de Rhodes puis celle de Malte nous enseignent la valeur qu'un port bien fortifié peut conserver pendant des siècles. C'est vrai, mais, d'une part, tous les ports ne sont pas bien fortifiés en Europe du Nord il s'agit souvent de plages et de baies plutôt que de ports bâtis en pierre ou en bois; d'autre part, même le port le plus sûr ne vaut que peu de chose si l'on ne peut plus en sortir, donc, pour être mobile, toutes les contingences maritimes entrent de nouveau en jeu.

Il est probable que ce sont ces contingences qui font que, pour nous autres contemporains, la thalassocratie est très difficile à saisir sur le plan fondamental, car notre culture, malgré toute la rhétorique autour de la mobilité et de la mondialisation, est en fin de compte très sédentaire. Nous avons du mal à comprendre ce »sens de l'espace« qui ne connaît pas de lieu. Notre »sens de l'espace« est celui qui commence avec les sencadrements` du Moyen Âge et que l'on doit, en premier lieu, à ce chef-d'œuvre mental que l'Église a créé en rendant l'espace constant et homogène. Même sans ces révolutions de l'imaginaire commun, déjà, sur le plan pratique, il est relativement aisé de se localiser sur la terre ferme, c'est-à-dire de savoir retrouver le grand orme de Gisors ou le lieu où convergent la Loire et le Cher. Nous savons que les marins, eux aussi, sont capables de telles prestations, mais cela demande beaucoup d'apprentissage et d'expérience. En outre, le »lieu« n'a que peu d'importance dans leur quotidien vécu et dans leurs cartes mentales, justement parce qu'ils dépendent beaucoup de ces contingences déjà évoquées, où l'on se rencontre, où l'on va, où l'on se retrouve. Il n'y a pas de »chez soi«, il n'y a pas d'»ailleurs«, pas au sens local. C'est pourquoi la thalassocratie proprement dite n'est pas imaginable géographiquement, et ceux qui la vivent ont une tout autre vision du monde et des rapports spatiaux. Il n'est même pas sûr que le titre de cet article, l'histoire politique des >espaces maritimes`, donne justice à ces visions toutes particulières; il est possible que l'espace ne se constitue pas comme étendue mais comme réseau.

Quant à nos deux frères danois, le roi sur terre et le roi sur mer, il faut désormais relire leur histoire à la lumière d'un autre récit, celui de Dudon de Saint-Quentin ${ }^{20}$. Ce premier des grands historiens des Normands avait une perspective du développement du pouvoir viking tout à fait différente. Selon Dudon, chanoine franc des alentours de l'an mil, et ses successeurs - Guillaume de Jumièges le bénédictin, puis au XII siècle Benoît le clerc de la cour angevine ${ }^{21}$-, les Vikings qui dévastaient les côtes franques n'étaient pas des héritiers enclins à se contenter d'un royaume mi-terrien mi-maritime. C'était ainsi que les Danois du XII ${ }^{\mathrm{e}}$ siècle se racontaient les débuts de leur propre pays. Les Francs, formant leur récit à la base des modèles anciens, se représentent l'activité

19 Pour les synthèses, voir Matthias PuHLE, Die Vitalienbrüder. Klaus Störtebeker und die Seeräuber der Hansezeit, Francfort/M. 1992; Olaf OLSEN, Ufredens hav. En 600 år gammel sørøverhistorie fra Østersøen, Copenhague 2002.

20 Dudon de Saint-Quentin, De moribus et actis primorum Normaniae ducum, éd. Jules LAIR, Caen 1865, en particulier chapitre I,1, p. 129.

21 Guillaume de Jumièges, Gesta Normannorum ducum, éd. Elisabeth VAN HouTs, Oxford 19921995; Benoît [de Sainte-Maure], Chronique des ducs de Normandie, éd. Carin FAHLIN, Uppsala $1951-1979$. 
maritime des gens du Nord comme résultat de la misère pure et simple. C'est de la polygynie débridée de ces païens barbares qu'il résulte une surpopulation endémique contre laquelle il n'y a d'autre remède que l'autodécimation. On tire au sort - procédé païen par excellence chez les chroniqueurs médiévaux - et ceux qui perdent sont condamnés à l'exil, ravageant désormais les côtes de la chrétienté. Naturellement, ils ne peuvent rien s'imaginer de plus beau que de trouver un paysage comme celui de l'embouchure de la Seine: »inculte et déserte, mais très fertile - il n'y faut que des hommes pour la prendre sous l'araire!«22 Ce récit du nouvel Énée marque toujours l'histoire d'une Normandie rapidement colonisée par des gens du Nord, qui étaient pressés de se laisser intégrer dans le monde franc: c'est pourtant une interprétation qui dépend de l'idée que, par principe, la vie maritime est déficitaire et provisoire.

Il serait pourtant téméraire d'écarter la vision >dudonienne`, le regard fixé sur la terre ferme tout entière, pour la remplacer par une histoire du Moyen Âge qui consisterait en deux moitiés égales, l'une continentale, l'autre maritime - enfin, une vision qui refléterait le partage du vieux roi Ro, car il se pourrait que pour les lecteurs de la chronique du $\mathrm{XII}^{\mathrm{e}}$ siècle, ce partage ait été juste. Or, il faut pourtant bien garder à l'esprit le contexte dans lequel l'épisode se trouve dans la chronique: c'est le passé païen raconté par un chanoine, un passé qui - semblable à ce qui se passe ailleurs en Europe avec la mise en valeur de l'histoire romaine - est prisé, mais qui appartient à l'époque d'avant la conversion; il ne reviendra donc plus jamais. Il y a une tendance archaïsante, peutêtre avec un flair de conservatisme, dans cette invention d'un propre passé. À l'époque où fut rédigée la Chronique, le Danemark était en plein essor agraire, et sa royauté, nouvellement affirmée aux dépens de plusieurs lignées concurrentes, poursuivait une politique résolument >européenneく, nouant des alliances avec la Saxe, l'Allemagne souabe puis la France 23 . En même temps, le chroniqueur anglo-normand Guillaume de Malmesbury nota au sujet du roi Édouard le Confesseur, qui avait vécu une soixantaine d'années auparavant, que celui-ci »se contenta du royaume qu'il avait hérité de ses ancêtres et renonça à la domination outre-mer (transmarinum imperium) comme chose trop laborieuse et trop barbare $\ll^{24}$.

Alors, la thalassocratie était-elle en train de passer de mode au cours du Moyen Âge? Peut-être conviendrait-il mieux de parler d'adaptation, de changement. Il est vrai

${ }_{22}$ Dudon de Saint-Quentin, De moribus (voir n. 20), p. 166: »terra haec penitus desolata [...] aratro non exercita [...] si fuerit frequentia hominum usitata, valde erit fertilis et uberrima...«. Il faudrait citer en intégralité ce cantique double sur la terre et la fille du roi franc que les chefs normands enjoignent à Rollon de >prendre « et féconder toutes les deux à la fois.

${ }^{23}$ En français, l'ouvrage de Thomas RIIS, Les institutions politiques centrales du Danemark 1100-1332, Odense 1977 (Odense university studies in history and social sciences, 46), est encore indispensable. La dernière mise au point d'ensemble est: Ole FENGER, Kirker rejses alle vegne 1050-1250, Copenhague 22002 (Gyldendal og Politikens Danmarkshistorie, 4).

24 Guillaume de Malmesbury, Gesta regum Anglorum, éd. Roger A. B. MYNORS, Rodney M. THOMPSON et Michael WINTERBOTTOM, Oxford 1998, ch. 259: »avitoque regno contentus transmarinum imperium ut laboriosum et barbarum despuit«. 
qu'il n'y aurait plus de >rois marins entières circonmarines comme ce >peuple de la mer du Nord $\iota$ des $\mathrm{V}^{\mathrm{e}}$, VI $\mathrm{VI}^{\mathrm{e}}$ et $\mathrm{VII}{ }^{\mathrm{e}}$ siècles dont nous percevons à peine les contours mais dont, à en croire les anthropologues, la culture était relativement uniforme à tous les bords de la mer, et nettement différente de celle des populations intérieures ${ }^{26}$. L'époque des Vikings, la première sur laquelle nous soyons assez bien informés, marque peut-être déjà un recul par rapport aux siècles antérieurs. Ainsi, l'opinion de Guillaume de Malmesbury, sinon celle de Dudon, à propos de la thalassocratie devenue trop `barbare`, pourrait de nouveau être intégrée dans le »grand récit« de l'essor de l'Europe postcarolingienne. Et tout comme dans le cas du Danemark, l'essor agraire du Moyen Âge central contribuait sans doute à une certaine marginalisation de l'Europe maritime, par rapport aux époques antérieures. Il faut dire qu'en aucun cas, tout au long du Moyen Âge, une formation politique ne s'est transformée en thalassocratie - tel que cela s'est produit plusieurs fois dans l'Antiquité et aux temps modernes. À notre avis, il y avait au Moyen Âge des cités comme Venise, ou un ensemble de cités comme la Hanse, qui étaient fortement thalassocratiques dès leur début, et d'autres pays, comme le Danemark et la Norvège qui perdirent leur caractère thalassocratique au cours du Moyen Âge central, pendant que la couronne d'Aragon passa d'une puissance pyrénéenne et espagnole à un empire maritime, sans pour autant perdre son caractère d'origine et son centre de gravité en Catalogne-Val d'Èbre. Cette tendance générale ne constitue pourtant pas tout le tableau. Il y aura toujours, même dans les circonstances nouvelles du Moyen Âge tardif, des phénomènes qui ressemblent beaucoup aux thalassocraties antérieures mais dont la signification et la place dans le contexte politique se sont modifiées. L'emprise toujours intensifiée des pouvoirs princiers sur le continent agraire n'atteignait les espaces maritimes que partiellement et par moments. Un contrôle suivi de la mer restait impossible, et le resserrement politique à terre ne se transférait pas à la mer. Il sera de première importance d'étudier le développement de la paix et du droit marin par rapport aux tendances semblables sur le continent - les similitudes, les décalages et les différences. Sans doute, le mouvement de la Paix de Dieu puis celui des princes depuis le XI ${ }^{\mathrm{e}}$ siècle n'avaient aucune équivalence en Europe maritime. Ce n'est qu'au moment où l'usage du droit et des lois s'est généralisé dans les royaumes que l'on voit, en Méditerranée puis dans la Baltique, surgir des tendances vers un droit commun maritime, encore tâtonnant et incomplet.

C'est le temps des hors-la-loi. La mer est pour ainsi dire la terre élue pour ceux qui sont »extra pacem positi «27, mis hors des communautés constituées par une paix qui revêt de plus en plus un caractère territorial. Le repli dans les terres sauvages, les bois et les montagnes, est un cul-de-sac. La mer, en revanche, offre des alternatives, elle accueille de nouveaux venus. "Qu'est-ce qui nous reste, enfin, sinon prendre la mer et

25 Voir Alfred P. SMYTH, Scandinavian Kings in the British Isles 850-880, Oxford 1977; Rory MCTURK, Studies in Ragnars saga loðbrókar and its major Scandinavian Analogues, Oxford 1991 (Medium aevum Oxford monographs, NS 15).

26 Voir Preben Meulengracht Sørensen, Saga og samfund, Copenhague 1977, p. 16-22.

27 Expression selon la donation du roi danois Cnut pour l'église de Lund, 1085, dans: Diplomatarium danicum, I 2, Copenhague 1963, $\mathrm{n}^{\circ} 21$. 
habiter parmi les ondes? « - c'est ce que disaient les Slaves victimes des ravages commis par les Saxons missionnaires et exacteurs d'impôts dans la chronique de Helmold de Bosau au milieu du XII ${ }^{e}$ siècle. »Quelle est notre faute si, chassés de la patrie, nous harcelons la mer ${ }^{28}$ ? « Grâce au terme romain bienvenu de "pirata«, les princes et leurs historiographes réussirent à faire reculer la concurrence thalassocratique autant que possible, pas seulement sur le plan politique et militaire, mais aussi sur le plan idéologique et mental. Or, l'histoire ultérieure de la piraterie, devenue trop fameuse sous son aspect des XVII ${ }^{e}$ et $\mathrm{XVIII}^{\mathrm{e}}$ siècles, nous fait facilement oublier que les thalassocrates du Moyen Âge tardif étaient souvent plus que de simples pirates. On pourrait plutôt les voir comme des représentants d'une culture politique complémentaire qui pouvaient très bien d'une manière ou d'une autre, exister en symbiose avec les formations terriennes. Bien souvent, ce ne sont justement pas les >desperados` qui deviennent les piratae les plus célèbres, les plus chanceux. C'est presque comme si, dans les luttes entre partis et factions aristocratiques, la mer s'offrait aux vaincus du jour comme zone de repli temporaire d'où ils pourraient ressortir le moment venu. De plus, on a souvent l'impression que la chasse à ce genre de participants au jeu politique, devenus thalassocrates par nécessité, ne se poursuit pas avec assez d'engagement de la part des vainqueurs. Ne représentent-ils pas toujours un pouvoir sur lequel on pourrait compter, de possibles futurs alliés? Les vrais ennemis, ceux qui ont perdu le jeu à tout jamais, seront tracassés et tués sans pardon. Ce n'est pas le cas des autres, momentanément mis hors jeu mais qui, dans des conditions différentes, pourraient un jour rentrer, constituant une sorte de ressource, voire une alternative politique. Il y a, dans l'histoire nordique tout comme dans celle de la Méditerranée, bon nombre d'exemples de cette complémentarité29.

Pour résumer, il existe deux acceptations possibles du terme, selon que l'on veut employer »thalassocratie« comme outil de la recherche en historie médiévale: l'une, appelée ici méthode descriptive, vise à faciliter l'approche comparative aux différents côtés de l'Europe maritime. Pour la Méditerranée, c'est l'occasion de sortir des discours >méditerranéistes` souvent quelque peu repliés sur eux-mêmes, de se détacher de son caractère unique du berceau de toutes sortes de civilisations et, avec toute la précaution requise, de carrefour des transferts culturels. Ce serait le comparatisme à la manière de Marc Bloch. L'autre acceptation, appelée ici méthode analytique, demanderait une approche bien plus étroite. Elle porterait sur les spécificités d'un pouvoir poli-

${ }^{28}$ Helmold de Bosau, Chronica Slavorum, éd. Bernhard SCHMEIDLER, Hanovre ${ }^{3} 1937$ (Monumenta Germaniae Historica, Script. rer. Germ. in us. schol., 32), chap. 84, p. 161: »Quid igitur restat, quam ut obmissis terris feramur in mare et habitemus cum gurgitibus? Aut quae culpa nostra si pulsi patria turbaveriomus mare...? «

29 Pour n'en citer qu'un: la Compagnie catalane, issue des événements autour de la conquête de la Sicile angevine par l'Aragon en 1283 et qui s'établit ensuite comme pouvoir marin quasi autonome en Égée, entre Byzance et les principautés franques de la Grèce; voir, entre autres, María Teresa FERRER i MALlol et al. (dir.), L'expansió catalana a la Mediterrània a la baixa edat mitjana, Barcelona 1989 (Anuario de estudios medievales/Anejos, 36); Peter Lock, The Franks in the Aegean 1204-1500, Londres 1995; David Agustí, Los Almogávares. La expansión mediterránea de la corona de Aragón, Madrid 2004; Ernest MARCOS HIERRO, Almogàvers: la història, Barcelone 2005. 
tique qui non seulement s'étend sur les espaces maritimes, mais qui s'y concentre et s'en contente. Ce serait, à notre avis, plutôt une enquête qui va dans le sens de l'appel à l'»anthropologie politique« que Jacques Le Goff a fait jadis ${ }^{30}$. Nous avons ainsi tenté ici d'initier le débat autour de ces questions ${ }^{31}$.

30 Notamment en intitulant la quatrième partie de son fameux recueil d'articles: L'imaginaire médiéval, Paris 1985, »Vers l'anthropologie politique«; l'expression s'y retrouve (p. 348) dans le cadre de son article »L'histoire politique est-elle toujours l'épine dorsale de l'histoire?«.

31 Avec le congrès »Medieval Thalassocracies: Kingdoms of the Northern Seas « qui s'est déroulé à l'université de Gotland, à Visby, en septembre 2005, suivi par un atelier au château de Gottorp à Schleswig en février 2008; dans le cadre du $48^{\mathrm{e}}$ Historikertag à Berlin en octobre 2010, j'ai organisé, conjointement avec Nikolas Jaspert, une section vouée aux thalassocraties médiévales. 